
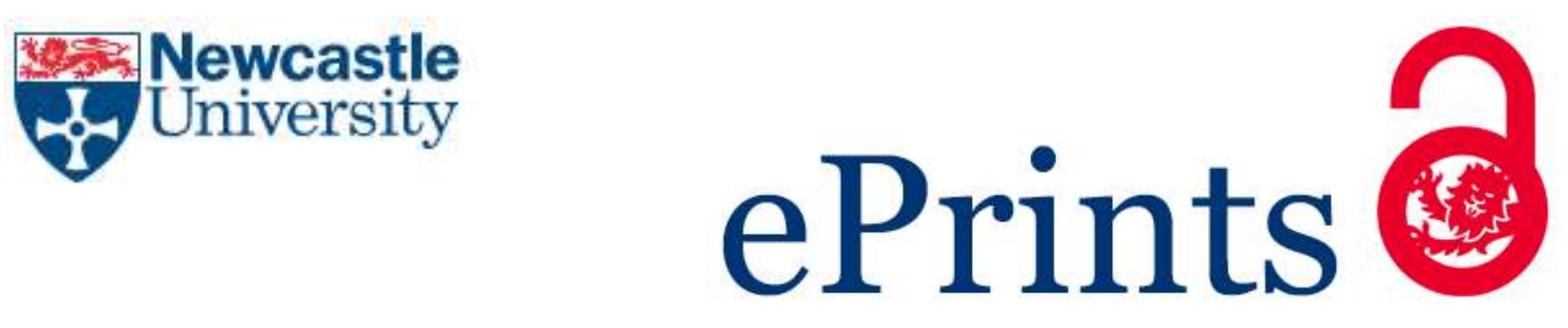

Lie MLS, Murtagh MJ, Burges Watson D, Jenkings KN, Mackintosh J, Ford GA, Thomson RG. Risk communication in the hyperacute setting of stroke thrombolysis: an interview study of clinicians. Emergency Medicine Journal 2015, 32(5), 357-363.

\title{
Copyright:
}

This article has been accepted for publication in Emergency Medicine Journal following peer review. The definitive copyedited, typeset version Lie MLS, Murtagh MJ, Burges Watson D, Jenkings KN, Mackintosh J, Ford GA, Thomson RG. Risk communication in the hyperacute setting of stroke thrombolysis: an interview study of clinicians. Emergency Medicine Journal 2015, 32(5), 357-363. is available online at:

http://dx.doi.org/10.1136/emermed-2014-203717

Date deposited:

$26 / 05 / 2016$

Embargo release date:

16 May 2017

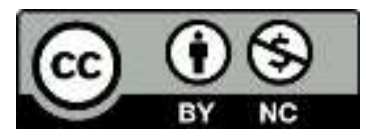

This work is licensed under a Creative Commons Attribution-NonCommercial 3.0 Unported License 


\section{Risk communication in the hyper-acute setting of stroke thrombolysis: an interview study of clinicians}

\section{Corresponding Author:}

Mabel L.S. Lie, PhD

Research Associate

School of Geography, Politics and Sociology

Newcastle University

Claremont Bridge Building

Newcastle upon Tyne

NE1 7RU

UK

Tel: +441912087510

Fax: +44 1912225421

E-mail: Mabel.Lie@ncl.ac.uk

\section{Names of Authors and affiliations:}

Lie, MLS ${ }^{1,2}$, Murtagh, $\mathrm{MJ}^{3}$, Burges Watson, $\mathrm{D}^{4}$, Jenkings, $\mathrm{KN}^{1}$, Mackintosh, $\mathrm{J}^{5}$, Ford, $\mathrm{GA}^{6,7}$, Thomson, $\mathrm{RG}^{5}$

${ }^{1}$ School of Geography, Politics and Sociology, Newcastle University, Newcastle upon Tyne, UK

${ }^{2}$ Institute of Cellular Medicine, Newcastle University, Newcastle upon Tyne, UK ${ }^{3}$ School of Social and Community Medicine, University of Bristol, Bristol, UK

${ }^{4}$ Wolfson Research Institute, Durham University, Durham, UK

${ }^{5}$ Institute of Health and Society, Newcastle University, Newcastle upon Tyne, UK ${ }^{6}$ Institute of Ageing and Health, Newcastle University, Newcastle upon Tyne, UK ${ }^{7}$ Oxford University Hospitals NHS Trust, Oxford, UK

Key words:

Stroke; thrombolysis; health communication

\section{Word Count:}

Abstract: 279

Main text without tables: 3617

References: 904 


\begin{abstract}
Objective: Communicating treatment risks and benefits to patients and their carers is central to clinical practice in modern healthcare. We investigated the challenges of risk communication by clinicians offering thrombolytic therapy for hyper-acute stroke where treatment must be administered rapidly to maximise benefit.

Method: Semi-structured interviews with 13 clinicians from three acute stroke units involved in decision making and/or information provision about thrombolysis. We report on clinicians' accounts of communicating risks and benefits to patients and carers. Framework analysis was employed.
\end{abstract}

Results: We identified the major challenges facing clinicians in communicating risk in this context i.e. disease complexity, patients' capacity and time constraints, and communicating quality of life after stroke. We found significant variation in the data on risks and benefits that clinicians provide and ways these were communicated to patients. Clinicians' communication strategies varied and included practices such as: a phased approach to communicating information, being responsive to the patient and family and documenting information they gave to patients.

Conclusion: Risk communication about thrombolysis involves complex uncertainties. We elucidate the challenges of effective risk communication in a hyper acute setting and identify the issues regarding variation in risk communication and the use of less effective formats for the communication of numerical risks and benefits. The paper identifies good practice such as the phased transfer of information over the care pathway, and ways in which clinicians might be supported to overcome challenges. This includes standardised risk and benefit information alongside appropriate personalisation of risk communication.

Practice Implications: Effective risk communication in emergency settings requires presentation of high quality data which is amenable to tailoring to individual patient's circumstances. It necessitates clinical skills development supported by personalised risk communication tools.

\title{
Section 1: What is already known about the subject
}

There is growing evidence that risk communication using established principles and access to patient decision aids facilitate better understanding and more informed decision-making. In the complex hyper-acute setting of stroke thrombolysis, Mangset et al (2009) have shown that patients have limited perceptions of the risks associated with stroke thrombolysis. To date, there has been no reported in depth research with clinicians exploring risk communication in this setting.

\section{Section 2: What this study adds}

This study provides results of a qualitative study examining doctors' reported approaches to risk communication about stroke thrombolysis in the complex and challenging setting of hyper-acute stroke. Apart from examples of good practice, we found variations identified in risk communication among clinicians that might be expected to adversely affect understanding by patients and their families. These findings identify the need for 1 ) greater appreciation of the evidence base of effective risk communication, 2) ready access to high quality evidence-based data in the form of information or decision support, and 3) clinical skills development. 


\section{Introduction}

Communicating the risks and benefits of treatments to patients is central to clinical practice in modern healthcare. It underpins informed consent and, arguably more importantly, creates the bridge between the evidence base for practice and effective decision making, particularly where decisions involve the patient [1 2 ]. Defined as the open two-way exchange of information between clinicians and patients on the risks and benefits of treatment, risk communication aims to facilitate better understanding and more informed decision-making [3 4]. The Cochrane Collaboration systematic review of effectiveness of patient decision aids (PtDAs) demonstrates that patients with access to such aids have improved risk perception [5 6]. Furthermore, The International Patient Decision Aid Standards (IPDAS) collaboration has included risk presentation as a core quality standard for PtDAs [7].

The evidence base for 'good' risk communication has generated discussion and critique. Issues raised include practice variation arising as a result of geographical location, professional specialisation, statistical literacy [8] and personal characteristics and values [9]. Understandings of risk and uncertainty can vary [9 10], with lay interpretations tending to be subjective compared to clinical presentations of population-based risk. Patient understandings of risk are prone to subjective interpretations which can vary from person to person and differ from clinician presentations of population-based risk based on scientific rationality [11 12]. Emotions too can play an important role in risk communication [13 14], which in turn is influenced by features of the clinical encounter [15]. Research involving trials and tests of probabilistic risk formats together with theory-building are limited [16]. Nevertheless, some established principles exist to enhance clarity [16 17] and strategies have been developed to better help patients understand risks [18-20]. These include consistency in the use of numeric formats and a clear reference class. The use of absolute risks, natural frequencies and consistent denominators is helpful, whilst use of relative risks has the potential to confuse rather than enlighten [16 17 21]. The use of pictographs (or icon arrays) has a growing evidence base for effectiveness [22].

Thrombolysis with recombinant tissue plasminogen activator (rtPA) is an effective early treatment for ischaemic stroke which must be given as soon as possible within a limited time window (up to four and a half hours) from onset of symptoms [23 24]. However there are risks of bleeding, particularly symptomatic intracranial haemorrhage $(\mathrm{SICH})$, which may lead to a worse outcome, including death, than might have occurred without treatment [25 26]. For clinicians, communicating risks and benefits to patients in this acute setting is complicated by the short time for decision making. Furthermore, stroke can manifest itself in a range of ways that are physically debilitating and mentally incapacitating, and may be disturbing to patients and their family [27 28]. An interview study with patients after stroke has shown that patients have limited perceptions of the risks associated with thrombolysis [29].

To date, this is the only primary in depth research with clinicians exploring risk communication in the context of a medical emergency. We describe doctors' reported approaches to risk communication about stroke thrombolysis in the complex setting of hyper-acute stroke, and discuss their implications. 


\section{Methods}

Semi-structured interviews were conducted with thirteen doctors (ten stroke consultants and one registrar, and two emergency physicians - see Table 1) from three acute stroke units that were study sites in a programme of research into hyper-acute stroke services [28]. The doctors were purposively sampled with respect to their involvement in decision making and/or information provision about thrombolysis with specific reference to involvement of patients and carers in decision making. Interviews lasted between thirty and sixty minutes. The topic guide (see Supplementary Box 1) was developed according to the aims of the research protocol and a literature review, and included questions about participants' attitude towards patient engagement, and the benefits and challenges of involving patients and carers in decision making and consent.

\section{Table 1: Clinicians across sites}

\begin{tabular}{lrrrr} 
& Site A & Site B & Site C & Totals \\
\hline Stroke Consultant & 4 & 2 & 4 & 10 \\
$\begin{array}{l}\text { Registrar } \\
\begin{array}{l}\text { Emergency Medicine } \\
\text { Consultant }\end{array}\end{array}$ & & & & 1 \\
\hline TOTALS & 2 & 7 & 9 & 2 \\
\hline
\end{tabular}

Clinician interviews conducted by DBW and MLSL were audio-recorded and transcribed verbatim. A five stage process of analysis was adopted involving: familiarisation with the data through reading and rereading the transcripts for recurrent themes; identification of a thematic framework based on the objectives of the research; a process of indexing in which transcripts were annotated with codes derived from the thematic framework; and both summarising and synthesising the data into charts that used representative quotes to demonstrate themes [30]. NVIVO data management software was used, and the findings were interrogated and validated in data clinics attended by MM, DBW, KNJ, JM and MLSL. For a clinical perspective and to counter the fragmentation and de-contextualisation of the data through the coding process [31], individual interviews were read in full by RT.

\section{The sites}

Of the three sites in our study, Site $\mathrm{C}$ had been one of the pioneer research centres for thrombolysis for more than ten years. Clinicians at Site $\mathrm{C}$ did not routinely use standardised risk estimates to quote to patients. Sites A and B, serving a large rural area as well as urban and suburban conurbations, had recently started to offer thrombolysis. A standardised protocol had been developed to support the delivery of their thrombolysis service. Their service included some provision of telemedicine services, with Emergency Medicine consultants referring to stroke consultants remotely for advice on whether to offer thrombolysis. The protocol included a standard script on the risks and benefits of treatment for Emergency Medicine doctors to use with patients. 


\section{Results}

The overall data analysis of the clinician interviews generated a total of three themes and 17 subthemes. The results related to risk communication were drawn from the following themes (and subthemes): clinician perspectives (difficulties professionals face; patient and carer responses); going about decision-making (capacity and consent; risk communication); and contextual features (acute stroke management).

\section{Understanding the problem}

Table 2: Understanding the problem:

\begin{tabular}{|c|c|}
\hline \multicolumn{2}{|c|}{ The problem of disease complexity } \\
\hline $1 a$. & $\begin{array}{l}\text { diagnosing stroke is not as easy as diagnosing MI, there's a lot of, yeah it's a bit } \\
\text { more tricky diagnosis (DocA01) }\end{array}$ \\
\hline $1 b$. & $\begin{array}{l}\text { I think again that's one difference in the areas of a stroke and heart attacks, a heart } \\
\text { attack yes you do have severe heart attacks and milder heart attacks but, um, in a } \\
\text { way there seems to be less variation compared to stroke. (DocB01) }\end{array}$ \\
\hline 1c. & $\begin{array}{l}\text { There's a similarity except with a stroke it's a higher risk treatment. There's a } \\
\text { slightly greater risk of side-effects and you know nasty complications (DocA05) }\end{array}$ \\
\hline $1 d$. & $\begin{array}{l}\text { Yeah, you have to give them some idea of what lies ahead. Again that's difficult } \\
\text { because some people will get worse, some people spontaneously do get better and } \\
\text { [you] even occasionally get caught out and that person who has a very severe } \\
\text { stroke actually does really well. But again you don't know that and you have to give } \\
\text { people an idea of what the impact is without the treatment (DocB01) }\end{array}$ \\
\hline $1 \mathrm{e}$. & $\begin{array}{l}\text { I am trying to help people accept that there may be some risks involved in that. But I } \\
\text { am not playing those up in all honesty. Because it seems to be from people's } \\
\text { reactions that they, they immediately, that eh, particularly families focus on that } \\
\text { immediately (DocA03) }\end{array}$ \\
\hline $1 f$. & $\begin{array}{l}\text { It was just coming up to } 3 \text { hours and err, I told the family that there was a risk of } \\
\text { bleeding, he was at higher risk of bleeding because em because he had diabetes and } \\
\text { whatever it was, his blood pressure was a bit high but he was going to be ... from } \\
\text { this stroke and err they were very keen to do anything they can. So he actually got, } \\
\text { I told them about risk of bleeding and despite the fact that } 2 \text { CTs [...] which showed } \\
\text { that he didn't have any bleeding at all actually he just took a massive brain swelling. } \\
\text { When he died the family turned around and said that we had killed him basically. } \\
\text { (DocC08) }\end{array}$ \\
\hline \multicolumn{2}{|c|}{ The problem of communication: capacity and time } \\
\hline 2a. & $\begin{array}{l}\text {....depending on what else is happening with them. You don't know whether they're } \\
\text { completely taking the information on board. (DocA01) }\end{array}$ \\
\hline $2 b$. & $\begin{array}{l}\text { Erm, the fact that people weren't expecting this to happen, are very shocked and } \\
\text { erm, sometimes can't really appreciate that you're saying that they've had a stroke } \\
\text { and they're at the moment severely disabled from it. Um, er hey they've got no } \\
\text { experience of what a stroke is. Most people tend to associate strokes with death } \\
\text { shortly afterwards (DocB01) }\end{array}$ \\
\hline 2c. & $\begin{array}{l}\text { you've just given them this whole raft of information about risks and possible side- } \\
\text { effects and this could happen and that could happen and you're almost pushing } \\
\text { them into a decision ... because you know you're under time pressure (DocA05) }\end{array}$ \\
\hline $2 \mathrm{~d}$. & $\begin{array}{l}\text { so in the context of thrombolysis you do actually have to say, we really can't wait } \\
\text { for that amount of time do you actually do something so, you are pressurising the } \\
\text { patient but you have to be honest about that (DocA07) }\end{array}$ \\
\hline
\end{tabular}




\begin{tabular}{|l|l|}
\hline 3a. & $\begin{array}{l}\text { I would say for example, the sort of construct we've had is particularly if you get a } \\
\text { younger patient say who's a musician or uses their hands at work and they've got a, } \\
\text { if they get even a mild stroke with a [ ] function they probably won't work again } \\
\text { (DocA02) }\end{array}$ \\
\hline 3b. & $\begin{array}{l}\text { You have to put it into the context of whether people are actually, you know, going } \\
\text { to be in their own homes, are they going to be able to look after themselves or are } \\
\text { they going to require a nursing home, making them more dependent on others, you } \\
\text { have to put it into that context rather than simply saying well their leg might not } \\
\text { get better (DocB01) }\end{array}$ \\
\hline 3c. & $\begin{array}{l}\text { Um, and then obviously if they've had something affecting their speech and that's } \\
\text { all it is then you, you, you would then focus very much on the impact of the speech, } \\
\text { but again that's tricky because then you're saying well, you know, how important do } \\
\text { you think it would be to them to be um, er, um exposed to the risk of this treatment } \\
\text { for the benefit of being able to talk better? Um, um so with more individual specific } \\
\text { impairments it is a bit trickier (DocB01) }\end{array}$ \\
\hline
\end{tabular}

\section{The problem of disease complexity}

Clinicians interviewed were very aware of the challenges of risk communication in the hyper-acute setting. Describing disease presentation, they gave accounts of borderline cases where balancing risks and benefits might be difficult for them, let alone the patients. Symptoms may fluctuate over the course of the time window for treatment, adding additional uncertainty. Older patients often suffer multiple co-morbidities and there were those who presented with no clear onset time. These factors complicated diagnosis, risk communication and informed consent. This heterogeneity of presentation further explains why thrombolysis in acute stroke is unique in contrast, for example, to that in acute myocardial infarction (see Table 2, 1a and b). Clinicians also referred to the higher risk of adverse effects of thrombolysis in stroke patients than in patients with MI (see Table 2, 1c).

Because of the nature of stroke, doctors reported facing challenges in predicting the likely outcome with thrombolysis for individual patients, given the probabilistic nature of treatment effectiveness (see Table 2, 1d and 1e). Even when the risks were explained and there is consent to treatment, serious adverse effects are difficult to accept (see Table 2, 1f). This element of uncertainty with regards to advice to individual patients presents challenges in communicating the probabilistic nature of risk.

\section{The problem of communication: capacity and time}

Clinicians highlighted the challenges of communicating with patients with limited capacity due to the stroke itself or existing co-morbidities such as speech and hearing impairments (see Table 2, 2a). They have to be alert to exactly how the stroke has affected the patient, particularly in terms of their ability to understand as well as to communicate.

Clinicians also recognised that there was an added layer of emotional response to the acute and often unpredicted stroke. Many patients associated stroke with mortality, therefore bringing with it a heightened sense of alarm. Several clinicians described the typical scenario they encountered with patients (see Table 2, 2b).

Participants also reflected on being pressed for time to deliver effective therapy. After a CT scan to determine if the stroke is ischaemic, hence confirming eligibility, patients 
were put under pressure to decide if they would consent to thrombolysis (see Table 2, $2 \mathrm{c}$ and $\mathrm{d})$.

\section{The problem of communicating quality of life after stroke}

In communicating risk, the issues that clinicians were concerned with went beyond risk and benefit based on categorical outcomes, to translating risk and benefit information into implications for the patient's quality of life after stroke. This is complicated by the heterogeneous nature of stroke, not only in terms of its initial severity but also with reference to the patient's health status preceding stroke, such as prior dependency. Furthermore, communication of risks and benefits needs to take account of what is important to the individual patient. For example, the implications of old upper limb impairments may be markedly different for a professional pianist and a retired woman (see Table 2, 3a). This and other contextual factors, such as living arrangements and the availability of family support, can have significant implications for the impact and importance of the likely outcome with and without treatment (see Table 2, 3b). Clinicians report difficulties taking account of the specific trade-offs between risk and benefit with respect to quality of life outcomes (see Table 2, 3c).

\section{Risk and benefit information provided by clinicians}

Table 3: Risk and benefit information provided by clinicians

\begin{tabular}{|c|c|}
\hline \multicolumn{2}{|c|}{ Variation and attempts at standardisation } \\
\hline $4 a$. & $\begin{array}{l}\text { And I think that a protocol, a tele-medicine protocol will end up simplifying yes or } \\
\text { no. That's not the model we're going for, we have a very, very intensively consultant } \\
\text { led service here (DocCO1) }\end{array}$ \\
\hline $4 b$. & $\begin{array}{l}\text { That time we can probably, we can have protocols in place and things that are you } \\
\text { know everything, everyone is going to be in the one place then it's easy enough to } \\
\text { say well em, the nurses do this, this and this when they come in and the doctor does } \\
\text { this, this and this but because it's now going here, there and everywhere you can't } \\
\text { really em, you've got three groups of doctors to try and educate and they change } \\
\text { over every few months so it's just impossible (DocC08) }\end{array}$ \\
\hline 4c. & $\begin{array}{l}\text { I mean the risk would be that I mean that for example last night there was an } \\
\text { infectious diseases registrar that was on call who I had never met before I think he } \\
\text { was new to the hospital so I can't imagine he had ever seen a patient, probably } \\
\text { hadn't seen a patient with a stroke for about three or four years much less } \\
\text { thrombolysed one (DocC08) }\end{array}$ \\
\hline $4 \mathrm{~d}$. & $\begin{array}{l}\text { I know currently that } 100 \text { people, em 30, } 3 \text { ratio is the one that most clinicians seem } \\
\text { to be using but perhaps not using in a consistent manner. At least for us they don't } \\
\text { seem to be. (DocC07) }\end{array}$ \\
\hline $4 e$. & $\begin{array}{l}\text { I think there needs to be em, err a minimum standard, standardised information } \\
\text { available based on what you believe is the right interpretation of the trial. We have } \\
\text { to remember that this is based on em, err limited number of randomised trials ..... } \\
\text { This is a particularly heterogeneous disease it cannot be applied to a single patient, } \\
\text { I think the predictions em in model could be designed but again I don't think it can } \\
\text { be predicted for an individual group of pa-, individual patients so...(DocCO7) }\end{array}$ \\
\hline $4 f$. & $\begin{array}{l}\text {.... we believe these are the kind of risks and benefits but you know it cannot be } \\
\text { predicted to the individual patient (DocCO7) }\end{array}$ \\
\hline $4 \mathrm{~g}$. & $\begin{array}{l}\text { we have got ... a very good, very short, four line brief of these are the risks, these } \\
\text { are the issues. So, I feel content that I have got a good grounding in it, that I } \\
\text { understand it but rather than blag the risk issue it is nice to have it there in front of } \\
\text { me (DocA08) }\end{array}$ \\
\hline
\end{tabular}




\begin{tabular}{|c|c|}
\hline $4 \mathrm{~h}$. & $\begin{array}{l}\text { So between ourselves, between the consultants who are doing it, we have got } \\
\text { standard information that we give and tailor according to the needs of the patient } \\
\text { but we're all quoting the same risks and benefits. Which is a good thing. (DocA02) }\end{array}$ \\
\hline \multicolumn{2}{|c|}{ Verbal and numerical variations } \\
\hline 5 a. & $\begin{array}{l}\text { the figures are standard but I will tell them that the treatment, the treatment has } \\
\text { been shown to be, to be beneficial in that it can dissolve the clot and it can reduce } \\
\text { people's chances of becoming disabled, of being disabled as a result of the stroke } \\
\text { but as with all things there are potential complications and one of those } \\
\text { complications is, is bleeding, deterioration at, of the stroke and even death, and I'll } \\
\text { give those quotes in percentages, I will give that quote both as a statement and } \\
\text { followed by percentages because not everybody understands percentages and then } \\
\text { again reinforcing that there potential benefits but there are potential complications } \\
\text { (DocA02) }\end{array}$ \\
\hline $5 b$. & $\begin{array}{l}\text { [I say:] So I need to explain to you what the risk and the benefits are. Now the } \\
\text { benefit is, very difficult to express but it's a matter of improving the survival a little } \\
\text { bit but more importantly improving the recovery so that you're less disabled as a } \\
\text { result of this stroke, and the chances of that being beneficial to you is that if we } \\
\text { treat a hundred people then thirty-two of them will benefit in this way. Now on the } \\
\text { other hand the drug is quite dangerous, it can sometimes cause bleeding and } \\
\text { sometimes can cause catastrophic bleeding leading anywhere including into the } \\
\text { brain. So it could make the situation much worse but the chances of that occurring } \\
\text { is if you treat a hundred people with this treatment about two or three will be } \\
\text { harmed in that way. (DocC01) }\end{array}$ \\
\hline 5c. & $\begin{array}{l}\text { I'd have a conversation along the lines of, you know, em, if we don't do anything, } \\
\text { you know, your chances of being dependent are this. If we give you thrombolysis, } \\
\text { then an extra } 14 \text { or } 16 \text { people will be independent as a result of the stroke and there } \\
\text { is a risk of } x \text { per cent and x per cent of bleeding. (DocA07) }\end{array}$ \\
\hline $5 \mathrm{~d}$. & $\begin{array}{l}\text { there's a few people who perhaps make a little bit of a recovery but not full and } \\
\text { then, there's no difference in the vast majority and then a very small minority who } \\
\text { actually, you probably die as a result of the treatment at the end of the day. } \\
\text { (DocB02) }\end{array}$ \\
\hline 5e. & $\begin{array}{l}\text { Well what I tell them is that em, in terms of the benefit they've got first of all they, } \\
\text { they've got roughly a thirty per cent or one third better chance of having a good } \\
\text { outcome than if they don't get treatment em, and that roughly one in between one } \\
\text { in ten and one in twelve people will have a dramatic improvement you know with } \\
\text { that sort of spectacular recovery but most, for most people that doesn't happen eh, } \\
\text { but for the rest you're talking more has a thirty per cent better chance for getting } \\
\text { them to a good outcome in other words being independent I explain that to them, } \\
\text { em, you know and being able to do everything for themselves pretty much. Em, and } \\
\text { then tell them about the risk of haemorrhage and which in terms of symptomatic } \\
\text { bleeding which is between sort of em three and five per cent but I usually explain } \\
\text { that in terms of em, you know a one in em, a one in twenty risk rather than you } \\
\text { know because people don't tend to understand percentages (DocC08) }\end{array}$ \\
\hline $5 f$. & $\begin{array}{l}\text { so I've tried various ways of describing that around em, you know the same } \\
\text { proportion of people overall will die with or without this treatment it's just that they } \\
\text { tend to die of different things if they are given the treatment they tend to die of } \\
\text { complications of the treatment rather than complications of the stroke, it doesn't } \\
\text { make any difference to the risk of dying overall but it does improve your chances if } \\
\text { you survive of having a good outcome. (DocA03) }\end{array}$ \\
\hline
\end{tabular}

\section{Variation and attempts at standardisation}

Different stroke units adopted standardisation of risk data depending on how stroke services were organised. Site $\mathrm{C}$, as a pioneer centre, depended less on standardised data as one clinician explained (see Table 3, 4a). But with the re-configuration of stroke 
services due to major physical restructuring, another noted the challenge of junior or inexperienced doctors communicating risks and benefits (see Table 3, 4b and 4c).

A clinician at Site $\mathrm{C}$ had undertaken an audit of his unit, and found that there was variation in the figures quoted by colleagues (see Table 3, 4d). Acknowledging the limits of trial data and difficulties in making predictions on individual cases because of the heterogeneity of stroke (see Table 3, 4e), he worked on producing a leaflet for patients. From his perspective, patients had the right to a minimum standard of information, but the limits of predictability also had to be made clear (see Table 3, 4f). He had drafted the following information:

“Approximately 32 out of 100 (or 1 in 3) patients treated with 'clot busting' medication will benefit from the treatment. The treatment benefit means that the patient will be left less disabled than if not treated. One in every 10 patients may make a full recovery from stroke with no disability. On the other hand, 3 in every 100 patients treated will be 'worse off' because of treatment related side effects of bleeding in the brain." (Site $C$ draft leaflet for patients)

In comparison, in the telemedicine context, clinicians at Site A referred to the following standardised script developed to support consistent communication as beneficial to their practice (see Table $3,4 \mathrm{~g}$ and $4 \mathrm{~h}$ ).

- There is a risk that the treatment will cause bleeding in the brain, causing a worsening stroke. This occurs in 7 out of 100 patients treated and is fatal in 2 of these.

- Despite this, overall the treatment is much more likely to help than to cause harm.

- Without treatment of 100 people with a stroke, 26 will survive with minimal or no disability - with treatment of 100 people with a stroke, 40 will survive with minimal disability.

(Site A standard information used for quoting to patients)

\section{Verbal and numerical variations}

Individual doctors reported very different approaches to communication of risks and benefits of thrombolysis, and how these approaches varied. In the majority of cases, risk communication consisted of a verbal description of the treatment effect, and the chances of survival, disability and bleeding complications. These explanations of risk and benefit commonly comprised a mix of both words and numbers (see Table 3, 5a and 5b). There were however potential problems in the way words were used. In a report of what he said to patients (see Table 3,5c), a clinician moved the referent in the sentence from the patient ('you') to stroke patients in general ('people') leading to potential ambiguity. Doctors also described the use of 'elastic terminology' such as 'a little bit' and 'minority' and 'majority' (see Table 3, 5d).

The way that numerical data were presented varied quite markedly. Clinicians used a mix of absolute numbers, percentages, fractions, ratios and differing denominators (see Table 3, 5c and 5e); some clinicians believed that not all patients were comfortable with percentages (see Table 3, 5a and 5e). Clinicians expressed concern about presenting figures on overall benefit (i.e. overall reduction in dependency with no overall effect 
on mortality) alongside the risks of early bleed (including risk of early death from severe haemorrhage). These latter risks are incorporated within the data on overall benefit, but some clinicians clearly felt that the risks of treatment needed to be presented separately (see Table 3, 5f).

\section{Communication strategies adopted by clinicians}

Table 4: Communication strategies adopted by clinicians

\begin{tabular}{|c|c|}
\hline \multicolumn{2}{|c|}{ Adopting a phased approach } \\
\hline 6a. & $\begin{array}{l}\text { you start with basically saying you know 'I think you've probably had a stroke', you } \\
\text { might give a little explanation as to what that is, well, most people don't really } \\
\text { know what a stroke is, they have some idea but maybe don't understand it and then } \\
\text { you start getting into the process and it's probably, it's round about then that you } \\
\text { say 'You know there is this treatment that is now available, this is how it works. To } \\
\text { do this we're going to have to get a scan first, or we're going to have to do that } \\
\text { fairly quickly' erm then you get them to the sort of scanning process, which in itself } \\
\text { will take } 15,20 \text { minutes and then you take it from there and again quite often we } \\
\text { get to that stage and find that we can't give thrombolysis because they've had a } \\
\text { bleed rather than an ischemic type stroke. (DocA05) }\end{array}$ \\
\hline \multicolumn{2}{|c|}{ Being responsive to the patient and family } \\
\hline 7a. & $\begin{array}{l}\text { Erm, there's various levels of it potentially in terms of engagement because you've } \\
\text { got those patients who want to know absolute all risks, benefits, down to, you } \\
\text { know, um, proportions of this, chances of that happening but, erm, that data often } \\
\text { isn't available in that level of detail and then you've got other patients who just say, } \\
\text { well they're not that interested in risks and benefit, they just want to know, what } \\
\text { you would do (DocB01) }\end{array}$ \\
\hline $7 \mathrm{~b}$. & $\begin{array}{l}\text { I try to start general and broad and to see whether people are taking that } \\
\text { information, you know, taking that information in, erm, and will give more, and I } \\
\text { get, then go into more detailed information but adjust my language and detail } \\
\text { according to how people are responding and you know their ability to take } \\
\text { information at that time and their educational level. (DocA02) }\end{array}$ \\
\hline 7c. & $\begin{array}{l}\text { where the discussion is with the family on behalf of the patient then that is often } \\
\text { more difficult eh, because families feel eh, eh, that, that, they're anticipating a } \\
\text { situation where the patient dies and where they feel that some of the, they've made } \\
\text { a decision that's led to that (DocA03) }\end{array}$ \\
\hline \multicolumn{2}{|c|}{ Checking and documenting information } \\
\hline $8 a$. & $\begin{array}{l}\text { you give them the information and you ask them to paraphrase it back to you so } \\
\text { like you can always say to them can you explain to me what the benefits of the clot } \\
\text { busting treatment are can you try to explain what I just said to you about the } \\
\text { benefits of the clot busting treatment and I think a lot of the time they're not have } \\
\text { as clue as to what you've said to them even though we did put it in their notes in } \\
\text { the most simplest language possible eh, so I already do that em, and obviously you } \\
\text { would ask them what do you understand is the hazard of this treatment what do } \\
\text { you understand is the benefit of it em (DocC08) }\end{array}$ \\
\hline $8 b$. & $\begin{array}{l}\text { I mean I think everybody should be documenting what they've told the patient in } \\
\text { terms of the risks because if the patient's relatives then turn around and say well } \\
\text { you didn't tell us about that we say well actually I did and I wrote it in the notes } \\
\text { what I said to you so em, the chances are they forget anyway but at least if you } \\
\text { write it in the notes its...(DocCO8) }\end{array}$ \\
\hline
\end{tabular}




\section{Adopting a phased approach}

One crucially important strategy that was reported in dealing with the challenge of time pressure was a phased approach to risk/benefit communication. Clinicians recognised that patients needed first to understand that they had had a stroke and what this meant. This prepares the patient for the definitive discussion of risks and benefits after the scan. This phased approach can take place all through the patient's care pathway, contributing to the patient's preparedness for making the final decision (see Table 4, 6a).

\section{Being responsive to the patient and family}

Several clinicians believed being responsive to patient needs was very important, reporting that patients were not always comfortable with making decisions solely on the basis of numbers and did not always base their decision-making upon them (see Table 4, 7a). They adjusted their risk communication, for example by simplifying information or providing detail in stages, all the while modifying their language in an interactive way according to their perception of the individual patient's needs or understanding (see Table 4, 7b).

Doctors also perceived that patients often depended on their family members for decision-making support. Communicating risk to family members can be challenging where the patient lacks capacity, requiring sensitivity to family members' positions of responsibility (see Table 4, 7c).

\section{Checking and documenting information}

One strategy that clinicians employed was to ask patients to recall in their own words what had been explained to them (see Table 4, 8a). As an additional safeguard, it was recommended that the information provided was documented (see Table $4,8 \mathrm{~b}$ ).

\section{Discussion and conclusion}

\section{Discussion}

Research into risk communication strategies in different healthcare contexts has yielded a range of results. While there is appreciation for personalised risk information in patients at risk of stroke and coronary heart disease, this did little to effect behaviour change [32], or to encourage attendance at screening tests [33]. In the context of clinical genetics [34], supportive counselling proved to be more effective than knowledge assimilation. Such contexts are very different to emergency or hyper-acute situations, where near on-the-spot decision-making requires optimum knowledge translation. A review of the literature found decision support interventions (DSIs) in emergency departments were associated with improved patient knowledge and satisfaction [35]. Discourse analysis of 'risk talk' conducted in varied health contexts such as antenatal and urology clinics has identified important contextual influences [11 15]. As highlighted in this study, these include specialist skills and time frames. But to date, no 
such studies have been conducted on clinicians' risk communication practices in the hyper-acute context of stroke.

As a timely contribution to this body of evidence, our interview study of clinicians involved in communicating the risks and benefits of thrombolysis in the hyper acute stroke setting has detailed the complexity and challenges involved. These include disease complexity, patient capacity and time pressure, and quality of life issues. Importantly, clinicians were taking account of patients' health status both prior to, and as a result of, their stroke. Explanations of past and predicted health state, and the potential impact on quality of life, are an important corollary to risk communication because they enable the potential impacts of treatment to be seen in the wider context. Clinicians thought deeply about the challenges of good risk communication in this complex, hyper-acute setting and used a range of approaches to address them. They communicated risks and benefits orally in a variety of formats i.e. both verbal and numerical, and often used a mix of absolute numbers, percentages, fractions and odds ratios. Unfortunately, approaches such as percentages which do not refer to the base rate or reference class (e.g. patients of the same age group) have been found less effective in communicating risk [16].

The variation reported in clinicians' risk communication, alongside the role of nonstroke specialists in communicating with patients, suggests that there is potential value in standardised risk and benefit information. At the same time, there is a tension between standardisation and the appropriate personalisation of risk communication. The need for flexibility and creativity in communicating risk was also reported, including adapting the communication to the particular circumstances of the individual patient as far as possible. Clinicians were concerned that a standard summary of aggregated trial-derived data may not apply to individual patients whose clinical characteristics might make them more or less likely to suffer an intra-cerebral bleed, for example. Ideally, we would have mechanisms of personalising risk and benefit based on patient characteristics, but this is only rarely available in a form that supports such personalisation [1 36].

Clinicians employed various strategies in their risk communication, varying and tailoring their approach in response to cues from the individual patient and/or family. Other reported strategies were to check that risks and benefits were understood and to document information. More importantly, clinicians were phasing the transfer of information over the care pathway, as studies of clinicians in other contexts have found [37]. Thus, our study has identified in detail the specific problems related to risk communication in the hyper-acute context of stroke, points to possible solutions and invites clinicians to reflect on their current practice.

\section{Limitations}

The limitations of the research are common to qualitative research in general. These include the small purposive sample size and geographical distribution, thus affecting the generalizability of the findings, the applicability of which depends on regional stroke management protocols. An accepted limitation of the research method is its 
reliance on participants' self-reported experiences and views rather than actual recordings of clinician-patient interactions, which in an emergency context could have ethical implications. The interviews were conducted by two different researchers with different backgrounds (geography and sociology) but the data was interrogated by several researchers, contributing to data validation. In addition, an artefact of the interview process arises from clinicians' assumptions of what is expected from them in the interview, which may have influenced their reported accounts. Nevertheless, there were corroborations in the findings from patient interviews [28].

\section{Conclusion}

Risk communication about thrombolysis is one of those medical interactions in which complex uncertainties are involved. The human element in responding to such uncertainty results in practice variation because of the influence of institutional contexts, personal characteristics and values, and lay perceptions of risk. Added to this is the impact of professional specialisation, competence and accountability. In this paper, we elucidate the challenges of effective risk communication in a hyper acute setting and identify the issues regarding variation in risk communication and the use of less effective methods for risk communication such as the use of elastic terminology, percentages and a mix of numerical formats that can be potentially confusing to patients. We provide examples of good practice such as the phased transfer of information over the care pathway and being responsive to the patient and family, and identify ways in which clinicians might be supported to overcome challenges. This includes standardised risk and benefit information alongside appropriate personalisation of risk communication. Thus the findings of this study point to the value of risk communication training for stroke clinicians, and standardised information, particularly for non-stroke specialists and for less experienced clinicians working in the hyper-acute context of stroke. The complexity in risk communication highlighted in this study and the literature points to the need for further research that includes randomised controlled studies of decision support tools, and the development and application of more theoretically-oriented perspectives [ $\left[\begin{array}{lll}8 & 9 & 16\end{array}\right]$.

\section{Practice implications}

Examples of good practice uncovered by the study include clinicians' attention to: the needs of the individual patient; his or her pre-stroke life and quality of life issues; and the phased transfer of information. However, the identified variations in risk communication might be expected to adversely affect understanding by patients and their families. This is by no means unique to the setting of thrombolysis, but arguably in the time constrained setting, the study has clearly shown that there is particular potential for greater appreciation of the evidence base for effective risk communication and for enhancing clinicians' skills in risk communication. Such communication is likely to depend upon accessibility of up-to-date, consistent and reliable data presented in a form that promotes effective communication. As in other emergency settings [35], ready access to high quality evidence-based data in the form of information or decision support, alongside skills development, would be likely to improve risk communication. 
In other clinical settings, individualisation or subgroup presentation of data has proven helpful [ $\left[\begin{array}{ll}1 & 2\end{array} 36\right]$, but this is not without its challenges. Likewise, there are questions to be asked about how the standardisation of risk communication in hyper-acute stroke might affect decision making and about how it would be possible to introduce a level of standardisation without losing the important context dependent and adaptive nature of current (reported) practice, translated to individual level information for the patient. Training in terms of using more appropriate and effective formats for the communication of numerical risks and benefits is also recommended.

\section{Acknowledgements}

We would especially like to thank the research participants and secretarial support from the Institute of Health and Society on this project.

\section{References}

1. Thomson R, Edwards A, Grey J. Risk communication in the clinical consultation. Clinical Medicine 2005;5(5):465-69

2. Edwards A, Hood K, Matthews E, et al. The Effectiveness of One-to-one Risk-communication Interventions in Health Care. Medical Decision Making 2000;20(3):290-97

3. Ahl AS, Acree JA, Gipson PS, et al. Standardisation of nomenclature for animal health risk analysis. Reviews of Scientific \&Technical Office, UK Government 1993;12(4):1045-53

4. Edwards A. Risk communication - making evidence part of patient choices. In: Edward A, Elwyn G, eds. Shared Decision-Making in Health Care: Achieving Evidence-based Patient Choice. Oxford: Oxford University Press, 2009:135-41.

5. O'Connor A, Wennberg J, Legare F, et al. Toward the 'tipping point': decision aids and informed patient choice. Health Affairs 26(3):716-25, 2007 May-Jun 2007;26(3):716-25

6. Stacey D, Bennett C, Barry M, et al. Decision aids for people facing health treatment or screening decisions. Cochrane Database of Systematic Reviews 2011; Issue 10. Art. No.: CD001431

7. Elwyn G, O'Connor A, Stacey D, et al. Developing a quality criteria framework for patient decision aids: online international Delphi consensus process. BMJ 2006;333(7565):417

8. Ghosh AK, Ghosh K. Translating evidence-based information into effective risk communication: current challenges and opportunities. Journal of Laboratory \& Clinical Medicine 2005;145(4):171-80

9. Politi MC, Han PKJ, Col NF. Communicating the uncertainty of harms and benefits of medical interventions. Med Decis Making 2007;27(5):681-95

10. Hampel J. Different concepts of risk - A challenge for risk communication. International Journal of Medical Microbiology 2006;296(Suppl 40):5-10

11. Adelsward V, Sachs L. Risk Discourse: Recontextualization of numerical values in clinical practice. Text 1998;18(2):191-210

12. Misselbrook D, Armstrong D. Thinking about risk. Can doctors and patients talk the same language? Family Practice 2002;19(1):1-2

13. Hemmerich JA, Elstein AS, Schwarze ML, et al. Risk as feelings in the effect of patient outcomes on physicians' future treatment decisions: A randomized 
trial and manipulation validation. Social Science \& Medicine 2012;75(2):36776

14. Slovic P, Finucane ML, Peters E, et al. Risk as Analysis and Risk as Feelings: Some Thoughts about Affect, Reason, Risk, and Rationality. Risk Analysis: An International Journal 2004;24(2):311-22

15. Linell P, Adelsward V, Sachs L, et al. Expert talk in medical contexts: Explicit and implicit orientation to risks. Research on Language and Social Interaction 2002;35(2):195-218

16. Lipkus IM. Numeric, Verbal, and Visual Formats of Conveying Health Risks: Suggested Best Practices and Future Recommendations. Med Decis Making 2007;27(5):696-713

17. Gigerenzer G. Reckoning with risk: learning to live with uncertainty. London: Penguin, 2003.

18. Fagerlin A, Zikmund-Fisher BJ, Ubel PA. Helping Patients Decide: Ten Steps to Better Risk Communication. Journal of the National Cancer Institute 2011;103(19):1436-43

19. Gigerenzer G, Edwards A. Simple tools for understanding risks: from innumeracy to insight. BMJ 2003;327(7417):741-44

20. Paling J. Strategies to help patients understand risks BMJ 2003;327:745-48

21. Akl E, Oxman A, Herrin J, et al. Using alternative statistical formats for presenting risks and risk reductions. Cochrane Database of Systematic Reviews 2011;Issue 3. Art. No.: CD006776

22. Galesic M, Garcia-Retamero R, Gigerenzer G. Using Icon Arrays to Communicate Medical Risks: Overcoming Low Numeracy. Health Psychology 2009;28(2):210-16

23. Ahmed N, Wahlgren N, Grond M, et al. Implementation and outcome of thrombolysis with alteplase 3-4.5 h after an acute stroke: an updated analysis from SITS-ISTR. The Lancet Neurology 2010;9(9):866-74

24. Lees KR, Bluhmki E, von Kummer R, et al. Time to treatment with intravenous alteplase and outcome in stroke: an updated pooled analysis of ECASS, ATLANTIS, NINDS, and EPITHET trials. The Lancet 2010;375(9727):1695-703

25. Wahlgren N, Ahmed N, Dávalos A, et al. Thrombolysis with alteplase for acute ischaemic stroke in the Safe Implementation of Thrombolysis in StrokeMonitoring Study (SITS-MOST): an observational study. The Lancet 2007;369(9558):275-82

26. Lansberg M, Albers G, Wijman C. Symptomatic intracerebral hemorrhage following thrombolytic therapy for acute ischemic stroke: a review of the risk factors. Cerebrovascular Diseases 2007;24(1):1-10

27. Faircloth CA, Boylstein C, Rittman M, et al. Constructing the Stroke: SuddenOnset Narratives of Stroke Survivors. Qual Health Res 2005;15(7):928-41

28. Murtagh MJ, Burges Watson DL, Jenkings KN, et al. Situationally-Sensitive Knowledge Translation and Relational Decision Making in Hyperacute Stroke: A Qualitative Study. PLoS ONE 2012;7(6):e37066

29. Mangset M, Berge E, Førde R, et al. Two per cent isn't a lot, but when it comes to death it seems quite a lot anyway: patients' perception of risk and willingness to accept risks associated with thrombolytic drug treatment for acute stroke. Journal of Medical Ethics 2009;35(1):42-46 
30. Ritchie J, Spencer L. Qualitative data analysis for applied policy research. In: Burgess RG, Bryman A, eds. Analyzing qualitative data. London; New York: Routledge, 1994:173-94.

31. Smith J, Firth J. Qualitative data analysis: the framework aproach. Nurse Researcher 2011;18(2):52-62

32. Powers BJ, Danus S, Grubber JM, et al. The effectiveness of personalized coronary heart disease and stroke risk communication. American Heart Journal 2011;161(4):673-80

33. Edwards A, Evans R, Dundon J, et al. Personalised risk communication for informed decision making about taking screening tests. Cochrane Database of Systematic Reviews 2006(4):CD001865

34. Edwards A, Gray J, Clarke A, et al. Interventions to improve risk communication in clinical genetics: Systematic review. Patient Education and Counseling 2008;71(1):4-25

35. Flynn D, Knoedler MA, Hess EP, et al. Engaging Patients in Health Care Decisions in the Emergency Department Through Shared Decision-making: A Systematic Review. Academic Emergency Medicine 2012;19(8):959-67

36. Thomson R. How can epidemiological studies help us to prevent stroke? The example of atrial fibrillation [Review]. Age and Ageing 2002;31(Suppl 3):916

37. Rapley T. Distributed decision making: the anatomy of decisions-in-action. Sociology of Health \& Illness 2008;30(3):429-44 